SIMPóSIO: Tópicos fundamentais para a formação e o desenvolvimento docente para professores dos cursos da área da saúde Capítulo VIII

\title{
Aprendizado baseado em problemas
}

\section{Problem-based learning}

\author{
Marcos C. Borges ${ }^{1}$, Silvana G. F. Chachá2, Silvana M. Quintana ${ }^{3}$, Luiz Carlos C. Freitas ${ }^{4}$, \\ Maria Lourdes V. Rodrigues ${ }^{4}$
}

\begin{abstract}
RESUMO
AAprendizagem Baseada em Problemas (ABP), introduzida no ensino de Ciências da Saúde na McMaster University, Canadá, em 1969, é uma proposta pedagógica que consiste no ensino centrado no estudante e baseado na solução de problemas, reais ou simulados. Os alunos, para solucionar esse problema, recorrem aos conhecimentos prévios, discutem, estudam, adquirem e integram os novos conhecimentos. Essa integração, aliada à aplicação prática, facilita a retenção do conhecimento. Portanto, a ABP valoriza, além do conteúdo a ser aprendido, a forma como ocorre o aprendizado, reforçando o papel ativo do aluno neste processo, permitindo que ele aprenda como aprender. A ABP oferece diversas vantagens, como o desenvolvimento da autonomia, a interdisciplinaridade, a indissociabilidade entre teoria e prática, o desenvolvimento do raciocínio crítico e de habilidades de comunicação, e a educação permanente. Porém, para a sua utilização são necessários investimentos em recursos humanos e materiais, além de um programa de capacitação de professores e alunos bem estruturado, que devem sempre ser considerados antes de sua implementação.
\end{abstract}

Palavras-chave: Educação, Pequenos grupos, Aprendizagem Baseada em Problemas

\section{Principais Pontos de Interesse}

- A Aprendizagem Baseada em Problemas (ABP) consiste no ensino centrado no estudante e baseado na solução de problemas.

- O currículo dos cursos que utilizam ABP geralmente é dividido em módulos temáticos, que são compostos de várias sessões e integram diversas disciplinas e o conhecimento básico e clínico.

- Para solucionar um problema, os alunos recorrem aos conhecimentos prévios, discutem, estudam, adquirem e integram os novos conhecimentos.

- AABP valoriza, além do conteúdo a ser aprendido, a forma como ocorre o aprendizado, reforçando o papel ativo do aluno neste processo, permitindo que ele aprenda como aprender.

- A ABP estimula o desenvolvimento de habilidades técnicas, cognitivas, de comunicação e atitudinais; o respeito à autonomia do estudante; o trabalho em pequenos grupos; e a educação permanente.
1. Departamento de Clínica Médica, 3Departamento de Ginecologia e Obstetrícia, 4Departamento de Oftalmologia, Otorrinolaringologia e Cirurgia da Cabeça e Pescoço, Faculdade de Medicina de Ribeirão Preto, Universidade de São Paulo

2. Departamento de Medicina, Universidade Federal de São Carlos
Correspondência: Profa Dra Maria Lourdes V Rodrigues

Av. Bandeirantes, 3900

CEP: $14049-900$

Ribeirão Preto/SP Email: mdlvrodr@fmrp.usp.br

Artigo recebido em 22/05/2014 Aprovado para publicação em 19/06/2014 
- O elemento central da ABP é o aluno, e o grupo tutorial é a base do método, que conta com a facilitação de um tutor.

- Inúmeras fontes podem servir como problemas para uma sessão de ABP, tais como casos descritos em papel, pacientes reais, pacientes simulados, exames laboratoriais, vídeos, áudios, textos de jornal ou revistas, fotos, artigos científicos.

- A implementação ou transição para um método de ensino como a ABP requer investimentos tanto em recursos humanos quanto materiais, além de um programa bem estruturado de capacitação de professores e alunos.

\section{Aprendizado baseado em problemas}

\section{História}

O princípio de que os seres humanos aprendem a partir de experiências do cotidiano, no qual se apresentam vários problemas que necessitam soluções, muitas vezes imediatas, foi a base do desenvolvimento do método "Aprendizagem Baseada em Problemas" (ABP, ou PBL do inglês "Problem Based Learning"). ${ }^{1}$

Através da obtenção, análise e síntese dos dados disponíveis, são identificadas lacunas do conhecimento, que precisam ser preenchidas. A aplicação desses novos conhecimentos, em conjunto com métodos de raciocínio dedutivo, compõe as bases para a solução do problema em foco. Esse tipo de abordagem leva o estudante a "aprender a aprender". ${ }^{2}$

Apesar de utilizado anteriormente por outras áreas do conhecimento, a ABP foi introduzida no ensino de Ciências da Saúde na McMaster University, Canadá, em 1969, sob a coordenação de Howard S. Barrows. As principais características do programa eram: ausência de disciplinas, integração de conteúdo e ênfase na solução de problemas. ${ }^{1,3,4}$ Assim, o método (desafiador, no início) levaria ao desenvolvimento no estudante de habilidades para dirigir o próprio aprendizado, de integração de conhecimentos, de identificação e exploração de novos temas, gerenciamento da sua educação permanente e capacidade de trabalhar em equipe. ${ }^{1,5}$ A estrutura curricular do novo curso era constituída por Unidades Interdisciplinares e no ano final, internato rotatório. As habilidades clínicas e de comunicação eram adquiridas em unidade vertical paralela. ${ }^{3}$
Depois da McMaster, várias escolas de Ciências da Saúde passaram a utilizar a ABP como base da estrutura curricular, de forma plena ou, inicialmente, como um currículo paralelo, ou em parte da grade curricular. Entre elas, estão: Maastrich University (Holanda), Southern Illinois School of Medicine (EUA), Faculté de Medicine - Université de Sherbrooke (Canadá) e Harvard Medical School (EUA). ${ }^{1}$ Estas, assim como outras instituições, oferecem oportunidades para treinamento e aperfeiçoamento de docentes de outras Universidades no método ABP. A Maastrich University tem exercido importante papel na divulgação da ABP no Brasil e atuado amplamente na formação de docentes brasileiros, de diversas universidades.

Os principais determinantes para a introdução de inovações no ensino de Ciências da Saúde são: avanços da ciência e da tecnologia para diagnóstico e tratamento e industrialização; mudanças de legislações; mudanças econômicas, políticas e sociais, com a consequente modificação do sistema de saúde e valorização da prevenção; surgimento de diferentes doenças; descoberta de novos medicamentos; e por pressões dos alunos e pela vontade de reitores, de diretores, de departamentos de ensino e de colegiados. ${ }^{6}$

Quando são fundadas novas escolas têm peso significante, também, as escolhas das agências de fomento e fundações que as apoiam financeiramente. Por exemplo, a fundação da FMRP-USP contou com o apoio da Fundação Rockfeller para o equipamento de laboratórios e aperfeiçoamento de professores. Isso influenciou no modelo de estrutura curricular, na estruturação departamental, no regime de trabalho dos docentes e na ênfase na geração de conhecimentos. Da mesma forma, diversas escolas médicas brasileiras adotaram o modelo $\mathrm{ABP}$ porque, nas últimas décadas do século XX e no início do século XXI, fundações apoiadoras da implantação de Escolas de Ciências da Saúde acreditaram no método e têm estimulado a sua utilização e apoiado a capacitação dos docentes.

Atualmente (2014) existem no Brasil 218 Escolas Médicas, $19 \%$ das quais declaram utilizar o método ABP7. Apesar da tendência atual de valorização do desenvolvimento gradual de competências profissionais em situações da vida real, que demandem conhecimento, habilidades e atitudes, o PBL continua sendo usado em grau maior ou menor, em muitas escolas ao redor do mundo. ${ }^{8}$ 


\section{O que é a Aprendizagem Baseada em Problemas (ABP)?}

A ABP é uma proposta pedagógica que consiste no ensino centrado no estudante e baseado na solução de problemas. O currículo dos cursos que utilizam ABP geralmente é dividido em módulos ou unidades temáticas, que são compostos de várias sessões e integram diversas disciplinas e o conhecimento básico e clínico.

O aprendizado ocorre a partir da apresentação de problemas, reais ou simulados, a um grupo de alunos. Os alunos, para solucionar este problema, recorrem aos conhecimentos prévios, discutem, estudam, adquirem e integram os novos conhecimentos. Essa integração, aliada à aplicação prática, facilita a retenção do conhecimento, que pode ser mais facilmente resgatado, quando o estudante estiver diante de novos problemas. ${ }^{1}$ Portanto, o método ABP valoriza, além do conteúdo a ser aprendido, a forma como ocorre o aprendizado, reforçando o papel ativo do aluno neste processo, permitindo que ele aprenda como aprender. ${ }^{9}$

A ABP estimula o estudante a desenvolver habilidades para gerenciar o próprio aprendizado, buscar ativamente as informações, integrar o conhecimento, identificar e explorar áreas novas, com isso o estudante adquire ferramentas para desenvolver habili- dades técnicas, cognitivas e atitudinais para a prática profissional e também para aprender ao longo da vida. ${ }^{1,10}$ Desta forma, a ABP caracteriza-se por fomentar a aprendizagem significativa, a articular os conhecimentos prévios com os de outros estudantes do grupo, a indissociabilidade entre teoria e prática, o respeito à autonomia do estudante, o trabalho em pequenos grupos, o desenvolvimento do raciocínio crítico e de habilidades de comunicação, e a educação permanente. ${ }^{10,11}$ Além disso, à medida que estimula uma atitude ativa do aluno em busca do conhecimento e não meramente informativa, como é o caso da prática pedagógica tradicional, a ABP caracteriza-se como uma metodologia formativa. ${ }^{12}$

\section{Funções dos integrantes da ABP}

O elemento central da ABP é o aluno, e o grupo tutorial é a base do método. No grupo tutorial, os alunos são apresentados a um problema, pré-elaborado por um conjunto de docentes, e, com a facilitação de um tutor, são estimulados a discutir e elaborar hipóteses. Esta situação motivadora nos grupos tutoriais leva a definição de objetivos de aprendizagem, que serão os estímulos para o estudo individual. ${ }^{13} \mathrm{~A}$ Tabela 1 resume os papéis dos participantes do grupo tutorial.

Tabela 1: Descrição dos papéis dos participantes do grupo tutorial




Grupo tutorial: são pequenos grupos, tradicionalmente compostos por oito a dez estudantes e um tutor. Dependendo do "modelo" de ABP e do número de estudantes, o grupo pode optar, a cada sessão, por eleger um coordenador e um secretário, sendo que esses papéis devem rodiziar entre os alunos nas diferentes sessões, de forma a propiciar que todos sejam coordenadores e secretários. O tempo de cada sessão pode variar de acordo com o número de integrantes e com o tipo de problema apresentado, no entanto, sessões muito extensas, com mais de três horas de duração, podem ser cansativas e levar à redução da atenção e da produtividade individual e do grupo. ${ }^{2}$ Quando o grupo é composto, é recomendável que todos os integrantes, juntos, estabeleçam regras claras de funcionamento, a fim de garantir o trabalho harmônico ao longo das sessões. ${ }^{2,14}$ Geralmente os pequenos grupos são rearranjados a cada módulo ou semestre, de modo que os estudantes aprendam a trabalhar com diversos colegas. Desta forma, o pequeno grupo facilita o processo de aquisição de conhecimentos e contribui de maneira significativa para o desenvolvimento de outros atributos na formação do aluno, entre eles: habilidades de comunicação, trabalho em equipe, solução de problemas, respeito aos colegas e desenvolvimento de postura crítica. ${ }^{14,15}$

Tutor: É um membro do corpo docente que participa de um grupo tutorial. Esta participação ocorre durante um módulo temático ou semestre. O tutor necessita ser treinado e conhecer de antemão os objetivos de aprendizado pretendidos para cada problema. Porém, não deverá impor estes objetivos, nem desvendá-los para os alunos, pois o processo de aprendizado é tão importante quanto o conhecimento em si. Suas principais atribuições são: estimular o processo de aprendizagem dos estudantes; estimular o trabalho do grupo e a participação dos estudantes; respeitar a opinião dos estudantes; detectar eventuais rivalidades, monopólios, inconformismos; detectar estudantes com problemas; fornecer feedback e realizar avaliações. ${ }^{14,16,17}$ Considerando que na ABP não existe transmissão de conhecimento como nos modelos tradicionais, o tutor não necessita ser especialista nos temas, tampouco é esperado que ele dê uma aula para os estudantes. Assim, a interferência do tutor deve ser a mínima necessária e preferencialmente na forma de perguntas, com intuito apenas de estimular a participação ativa de todos estudantes e também corrigir rumos quando a discussão se afastar muito do tema proposto.
Estudante coordenador: é um estudante do grupo que deverá auxiliar a facilitação durante a discussão no grupo tutorial.

Estudante secretário: é um estudante do grupo que realizará as anotações referentes à discussão, garantindo que as várias etapas da discussão sejam anotadas de forma que o grupo não se perca na discussão e não volte a pontos que já foram discutidos anteriormente.

Demais estudantes: deverão se esforçar para realizar uma boa discussão do problema, de forma metódica, respeitando as diretrizes do coordenador do grupo.

Além dos grupos tutoriais, outras atividades e profissionais também são necessários na $\mathrm{ABP}$, como o treinamento de habilidades, aulas práticas, estágios, consultorias com especialistas, entre outras, a fim de complementar a formação dos estudantes.

\section{Passos de um grupo tutorial}

O grupo tutorial, geralmente, se desenvolve em sete passos, descritos na Tabela 2 e abaixo. ${ }^{17}$

\section{Tabela 2: Passos de um grupo tutorial}

1. Leitura do problema, identificação e esclarecimento de termos desconhecidos.

2. Identificação dos problemas propostos.

3. Formulação de hipóteses ("brainstorming”).

4. Resumo das hipóteses.

5. Formulação dos objetivos de aprendizagem.

6. Estudo individual dos objetivos de aprendizagem.

7. Rediscussão do problema frente aos novos conhecimentos adquiridos.

\section{Primeira sessão em grupo}

A dinâmica da sessão em grupo se inicia com a apresentação do problema, o qual pode ser simulado ou real. Dependendo do material utilizado, pode-se eleger um dos estudantes para apresentar o problema ou todos estudam a situação de forma individual. Nessa etapa, devem ser identificados possíveis termos desconhecidos, sendo o significado destes elucidado pelo grupo rapidamente ou levado à problematização. ${ }^{2,4,14}$ 
Após, o grupo deve identificar os problemas a serem discutidos e examiná-los de forma aprofundada o suficiente para delimitar os objetivos da sessão. A discussão segue com a intenção de explicar os problemas, utilizando conhecimentos previamente adquiridos e experiências de vida. Esta etapa tem sido denominada "brainstorming", uma vez que os estudantes são encorajados a explicitar todas as associações e significações. ${ }^{3,4}$

Após o "brainstorming", o grupo deve realizar uma síntese da discussão para facilitar a organização das ideias e a exposição dos limites de conhecimento, a fim de partir para a etapa de construção de hipóteses sobre a natureza do problema. Esta síntese pode ser feita pelo estudante-secretário, tendo o auxílio dos demais membros do grupo. As hipóteses geradas pelo grupo devem ilustrar explicações com base em mecanismos, de forma a entender os conceitos, evitando explicações simplificadas e superficiais. A participação de todos na construção das hipóteses é essencial, pois cada um dos estudantes deve se identificar com o produto da discussão em grupo, a fim de encontrar o estímulo para a etapa de estudo individual. ${ }^{2,14}$

A partir das hipóteses desenhadas, o grupo deve delimitar os objetivos de aprendizagem, o que pode ser feito na forma de questões. A elaboração das questões de aprendizagem deve refletir toda a discussão realizada nas etapas anteriores, novamente privilegiando o entendimento completo dos conceitos e mecanismos, do "como" em detrimento do "qual". O tutor deve se certificar de que todos os estudantes tenham clareza dos objetivos delimitados pelas questões de aprendizagem antes do final da sessão. ${ }^{4}$

De posse das hipóteses e questões de aprendizagem elaboradas na sessão, os estudantes partem para a etapa de estudo individual. A grade curricular deve incluir espaços para a realização desta etapa e a escola médica deve garantir o acesso a bibliografias variadas. Faz parte da metodologia ativa de ensino-aprendizagem tornar os estudantes aptos à pesquisa bibliográfica qualificada. ${ }^{2,14,18}$

\section{Segunda sessão em grupo}

No re-encontro do grupo após o estudo individual, os estudantes irão explicitar o produto de suas pesquisas de forma contextualizada, aplicando os novos conhecimentos à resolução das questões elaboradas e à elucidação dos problemas levantados na primeira sessão. É importante que o façam na forma de síntese, elaborada pelo próprio estudante, citando a bibliografia consultada, porém evitando a simples leitura dos textos científicos. Os componentes do grupo devem ser estimulados a ouvir e entender as ideias trazidas pelos colegas, de forma a complementar suas sínteses individuais por meio da socialização do conhecimento e da ajuda mútua. ${ }^{2} \mathrm{O}$ tutor deve estimular a análise crítica tanto da fonte bibliográfica utilizada, como da própria informação trazida, bem como sua aplicação à situação em discussão. ${ }^{14}$ Os estudantes devem ainda ter em mente que os novos conhecimentos adquiridos podem ser aplicados a diferentes situações e contextos. ${ }^{3,10}$

\section{Avaliação do processo ensino-aprendizagem}

Ao final de cada sessão em grupo é importante que seja aberto espaço para a reflexão e avaliação do processo de trabalho, feito na forma de auto-avaliação, avaliação dos colegas e do tutor. Cada membro do grupo deve ter espaço para sua avaliação sem interrupções ou réplicas. O ambiente precisa ser de respeito e cooperação, em busca do melhor funcionamento do grupo e melhor aproveitamento de cada um dos membros. Este momento permite sanar disfunções e dificuldades de relacionamento surgidas no grupo ao longo do processo. ${ }^{2,10}$

\section{Construção de problemas}

Os problemas na $\mathrm{ABP}$ constituem o ponto de partida, os "gatilhos", para a discussão e, consequentemente, o aprendizado. Desta forma, a qualidade dos problemas influencia o desenvolvimento do grupo e dos estudantes.

Teoricamente, inúmeras fontes, tais como casos descritos em papel, pacientes reais, pacientes simulados, exames laboratoriais, vídeos, áudios, textos de jornal ou revistas, fotos, artigos científicos, entre outras, podem servir de problema para uma sessão de ABP. Porém, todos os tipos de problemas necessitam ser relevantes e devem promover a discussão e estimular os estudantes a aprender durante o grupo e individualmente. ${ }^{19}$ Adicionalmente, mudanças no formato dos problemas estimulam o engajamento dos estudantes ao longo do curso.

Considerando que os estudantes utilizam seu conhecimento prévio nas discussões, algumas características, listadas na Tabela 3, devem ser lembradas durante a construção de um problema. 
Tabela 3: Características necessárias para a construção de problemas ${ }^{12,17,18,19}$

- Fácil leitura e adequados ao nível de conhecimento do grupo.

- Relevantes e, de preferência, conter situações que os estudantes enfrentarão em sua vida profissional.

- Promover uma integração do conteúdo básico e clínico.

- Estimular a discussão e o aprendizado de um número limitado de itens.

- Conter disparadores (pistas) para ativar o conhecimento prévio e guiar os estudantes durante a discussão.

- Não devem se muito concisos ou muito amplos.

- Não devem conter pistas escondidas, ser muito simples, muito complexos ou conter inúmeros distratores.

\section{ABP - Vantagens e Limitações}

Alguns estudos têm comparado currículos que utilizam ABP e o ensino médico tradicional. ${ }^{20,21} \mathrm{Em}-$ bora muitos autores não tenham encontrado diferenças substanciais entre os métodos, acredita-se que a ABP ofereça vantagens que só poderão ser constatadas com a evolução profissional do egresso, especialmente as características ligadas a autonomia, ao autoditatismo e ao desenvolvimento de uma postura profissional de base científica. Ademais, o protagonismo dos estudantes no processo de aprendizagem é um importante fator motivacional, levando a busca ativa do conhecimento e gerando um aprendizado mais eficaz. ${ }^{22}$

A interdisciplinaridade é outra importante vantagem da ABP sobre o ensino tradicional. A substituição de conhecimento fragmentado, oferecido em disciplinas, por situações reais, que envolvam vários aspectos do conhecimento, favorece uma aprendizagem significativa, contextual e, ainda, promove a integração dos conteúdos curriculares dos ciclos básico e clínico. Além disso, favorece a inserção dos estudantes em atividade de prática clínica já no início do curso médico, em conformidade com as principais diretrizes curriculares para o ensino médico.

A ABP favorece o desenvolvimento de habilidades de comunicação para trabalho em pequenos grupos, exposição de ideias, capacidade de argumentação e crítica. O respeito às diferentes opiniões, a autocrítica, o senso de responsabilidade, a capacidade de gerenciar projetos e as atividades de um grupo de trabalho também são importantes ganhos.
Os professores que atuam em um modelo de $\mathrm{ABP}$ não têm, como no método tradicional, total controle sobre o conteúdo desenvolvido no pequeno grupo.

Além disso, o aprendizado na ABP é centrado no estudante, não havendo transmissão de conhecimento, como no modelo tradicional. Isso pode causar um certo desconforto em professores que não foram bem treinados para a ABP.

Esse novo papel do professor no processo de ensino-aprendizagem tem sido uma das grandes limitações para a adoção da ABP nas escolas médicas, especialmente naquelas que se fundamentaram e se desenvolveram dentro do ensino tradicional. A dificuldade em se institucionalizar uma inovação, como uma nova abordagem metodológica, impossibilita a sua adoção. Mesmo os estudantes podem-se mostrar resistentes às mudanças. A quebra da passividade dos estudantes na aquisição do conhecimento gera desconforto e requer uma postura proativa, o que nem sempre é bem assimilado e aceito por todos os estudantes. ${ }^{23}$ Além disso, a falta de experiência de professores e estudantes com os fundamentos teóricos e práticos da $\mathrm{ABP} \mathrm{e}^{\prime}$ outro fator que limita a sua aplicação.

A implementação ou transição para um método de ensino como a $\mathrm{ABP}$ requer investimentos tanto em recursos humanos quanto materiais. $\mathrm{O}$ trabalho em pequenos grupos, naturalmente, eleva o tempo de atividade dos professores com os alunos e com isso faz-se necessária uma ampliação do corpo docente. Quanto aos recursos materiais, há necessidade de maior investimento para que sejam disponibilizados aos estudantes os mais variados recursos educacionais como bibliotecas, laboratórios, salas de estudo, recursos audio-visuais, e de informática, acesso livre a base eletrônica de dados, entre outros, uma vez que a ABP pressupõe autonomia do estudante na busca do conhecimento, e a estrutura para essa atividade precisa ser garantida pela escola. Além disso, é imprescindível que haja o desenvolvimento de um programa de capacitação de professores e alunos, para que se identifiquem e familiarizem-se com o novo modelo pedagógico. ${ }^{14}$

Finalmente, deve ser ressaltado que as vantagens e limitações da ABP devem ser analisadas, considerando-se, ainda, as condições específicas de cada currículo. Assim, para avaliar o efeito de um novo método sobre a formação do egresso torna-se imperativo analisar o currículo proposto e os subsequentes ganhos de aprendizagem. 


\begin{abstract}
The Problem-Based Learning (PBL), introduced at McMaster University School of Medicine, Canada, in 1969 , is a student-centered pedagogical approach based on critical thinking and problem solving. In order to solve a problem, students use their prior knowledge, discuss, study, acquire and integrate new information. This learning integration, combined with a practical application, facilitates knowledge retention. Therefore, students become actively involved in the learning process and improve the capacity of learn how to learn. PBL has several advantages, such as, it stimulates the development of autonomy on learning; interdisciplinary; integration of knowledge and practice; development of teamwork, critical thinking and communication skills; and continuing education. However, to be able to implement PBL on curriculum, it's necessary investments in human and material resources, including a well-structured training program for teachers and students.
\end{abstract}

Key-words: Education, Problem-Based Learning, Active Learning

\section{Referênclas Blbllográflcas}

1. Rodrigues MLV, Figueiredo JFC. Aprendizado centrado em problemas. Medicina (Ribeirão Preto). 1996;29:396-402.

2. Barrows HS, Tamblym RM. Problem-based learning: an approach to medical education. New York: Springer Publishing Company; 1980.

3. Lee RMKW, Kwan CY. The use of problem-based learning in medical education. J Med Education. 1997;1:149-57.

4. Barrows HS. The tutorial process. Springfield: Southern Illinois School of Medicine; 1988.

5. Bate E, Taylor DC. Twelve tips on how to survive PBL as a medical student. Med Teach. 2013;35:95-100.

6. Rodrigues MLV. Inovações no ensino médico e outras mudanças: aspectos históricos e na Faculdade de Medicina de Ribeirão Preto-USP. Medicina (Ribeirão Preto). 2002;35:231-5.

7. Nassif ACN. Escolas Médicas do Brasil http:// www.escolasmedicas.com.br/metodo.php2014 [cited 2014].

8. Bate E, Hommes J, Duvivier R, Taylor DC. Problem-based learning (PBL): getting the most out of your students - their roles and responsibilities: AMEE Guide № 84. Med Teach. 2014;36:1-12.

9. Toledo-Júnior ACC, Ibiapina CC, Lopes SCF, Rodrigues ACP, Soares SMS. Aprendizagem baseada em problemas: uma nova referência para a construção do currículo médico. Rev Méd. Minas Gerais. 2008;18:123-31.

10. Gomes R, Brino RF, Aquilante AG, Avó LRS. Aprendizagem baseada em problemas na formação médica e o currículo tradicional de medicina: uma revisão bibliográfica. Rev Bras Educ Méd. 2009;33:444-51.

11. Siqueira-Batista R, Siqueira-Batista R. Os anéis da serpente: a aprendizagem baseada em problemas e as sociedades de controle. Ciênc. saúde coletiva. 2009;14:1183-92.
12. Sakai MH, Lima GZ. PBL: uma visão geral do método. Olho Mágico. 1996;2:24-30.

13. Thomson JC. PBL-uma proposta pedagógica. Olho Mágico. 1996;2:7.

14. Wood DF. Problem based learning. BMJ. 2003;326(7384):32830.

15. Bligh J. Problem-based learning in medicine: an introduction. Postgrad Med J. 1995;71(836):323-6.

16. Sobral DT. Aprendizagem baseada em problemas: efeitos no aprendizado. Rev Bras Educ Méd.18:61-4.

17. Berbel NAN. "Problematization" and problem-based learning: different words or different ways? Interface Comun Saúde Educ. 1998;2:139-54.

18. Davis MH. AMEE Medical Education Guide No. 15: Problembased learning: a practical guide. Med Teach. 1999;21:13040

19. David D, Patel L, Burdett K, Rangachari P. Problem-Based Learning in Medicine: Royal Society of Medicine Press Ltd; 1999.

20. Kjærsdam F, Enemark S. The Aalborg experiment project innovation in university education: Aalborg Universitetsforlag; 1994.

21. Edmunds S, Brown G. Effective small group learning: AMEE Guide № 48. Med Teach. 2010;32:715-26.

22. Ribeiro LRC, Mizukami MGN. Problem-based learning: a student evaluation of an implementation in postgraduate engineering education. Eur J Eng Educ. 2005;30:137-49.

23. Gil AC. Metodologia do ensino superior: Atlas; 1990. 